Europhys. Lett., 41 (4), pp. 461-465 (1998)

\title{
Convective instability in foam drainage
}

\author{
S. Hutzler, D. Weaire and R. Crawford \\ Department of Physics, Trinity College - University of Dublin, Dublin, Ireland
}

(received 9 October 1997; accepted in final form 17 December 1997)

PACS. 82.70Rr - Aerosols and foams.

PACS. $47.60+\mathrm{i}-$ Flows in ducts, channels, nozzles, and conduits.

PACS. 47.20Ky - Nonlinearity (including bifurcation theory).

\begin{abstract}
The use of a column of steadily drained foam as a sample of wet foam is limited by flow instability as the liquid fraction is increased. We report a study of this instability, which takes the form of a convective roll of bubble motion at a value of liquid fraction roughly half that of the wet foam limit at which a static wet foam becomes unstable.
\end{abstract}

Introduction. - A simple experimental set-up where equal-size soap bubbles are introduced into a glass cylinder and subsequently wetted from top with soap solution can be used for a number of intriguing experiments. Bubbles with diameter of the order of the tube diameter crystallise to form ordered foam structures [1]-[3]. Bubbles that are small compared with the tube diameter have been used to study foam drainage, including the occurrence of a solitary wave [4], [5]. Under conditions of steady drainage with constant input of solution at the top of the column, the liquid fraction is approximately constant, providing a convenient experimental sample of wet foam, albeit not strictly in equilibrium.

This has been a convenient way of measuring the dependence of electrical conductance and capacitance, as functions of the liquid fraction [6], [7]. However, such measurements have so far been limited to liquid fractions below $\Phi_{1} \simeq 0.15$, to be compared with the extreme wet limit, which is approximately $\Phi_{1} \simeq 0.36$, because of flow instability. In the present letter we examine this instability, which is analogous to the transition from laminar to turbulent flow in an ordinary liquid. The observed large-scale flow pattern is also reminiscent of the sedimentation properties of monodisperse spherical polystyrene spheres in water [8]. In our experiment the initial instability takes the form of a slow convective roll of bubble motion over the entire column, as in fig. 1.

For low values of $\Phi_{1}\left(\Phi_{1} \leq 0.05\right)$, the individual cells have polyhedral shape; the foam is called "dry" or "Polyederschaum". In a foam with increased liquid fraction, the cell edges are rounded off, and the bubbles resemble more and more a packing of spheres ("wet" foam or "Kugelschaum"). Beyond some critical liquid fraction $\Phi_{1}^{\mathrm{c}}$, these spheres come apart, the foam loses its mechanical stability and becomes a bubbly liquid. This loss of stability of a foam has been studied numerically in two and three dimensions [9]-[12]; it has been called the "rigidity loss transition". In two dimensions $\Phi_{1}^{\mathrm{c}}$ has been identified as the density of a random packing

(C) EDP Sciences 


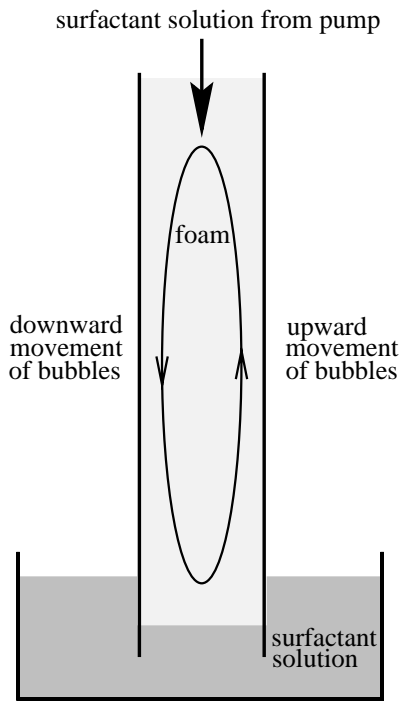

Fig. 1

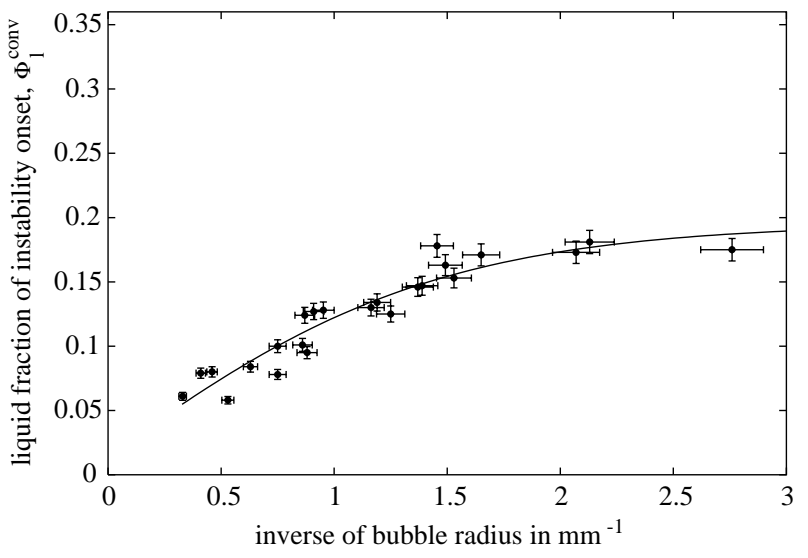

Fig. 2

Fig. 1. - Sketch of convective bubble motion in forced-drainage experiments. This dynamic instability occurs at liquid fractions that are well below the values for the rigidity loss transition.

Fig. 2. - Liquid fraction of the instability onset $\Phi_{1}^{\text {conv }}$ as a function of the inverse bubble radius. Note that all plotted data was taken for a foam column with diameter $1.5 \mathrm{~cm}$ and length $12 \mathrm{~cm}$. The solid line may serve as a guide to the eye.

of hard discs, leading to $\Phi_{1}^{\mathrm{c}} \simeq 0.16[9]$. In three dimensions the analogy is with a random packing of spheres (Bernal packing) for which $\Phi_{1}^{\mathrm{c}} \simeq 0.36$. (Both values apply only if the size distribution is not too broad.)

Experimental studies of the rigidity loss have so far only been reported for emulsions, which have similar mechanical properties to foams but generally have a much smaller structural scale [13]-[15].

Experimental observations. - The observations to be reported relate to the critical value of $\Phi_{1}$ for convective motion, that is, the particular value of the liquid fraction at which we observe an onset of a collective bubble motion. Roughly half of these bubbles move downward the tube with a constant velocity, while the other half moves upwards; see fig. 1.

Bubbles of equal size are produced by blowing $\mathrm{N}_{2}$ gas through a fine nozzle into a surfactant solution, and allowed to rise to fill a glass cylinder. Once the tube is filled with foam, the gas input is switched off and a steady flow of surfactant solution is added on top of the foam. By measuring the total height of the foam column and its immersion into the pool of liquid, its liquid fraction can be determined using the principle of Archimedes.

All our experiments were performed using ordinary dishwasher solution. We used glass tubes with diameters ranging from $10-20 \mathrm{~mm}$ and lengths $12 \mathrm{~cm}$ and $30 \mathrm{~cm}$, respectively. Bubble size was varied for different runs of the experiment by adjusting the gas pressure and/or using different nozzles. We used bubbles in the range $0.20 \mathrm{~mm}^{3}$ to $112.0 \mathrm{~mm}^{3}$. Their size was determined by filling fine capillary tubes in which the bubbles form ordered structures.

Surfactant solution is added to the foam from the top by use of a peristaltic pump with an adjustable flow rate up to $0.45 \mathrm{ml} / \mathrm{s}$. The input nozzle nearly touches the foam in order to 


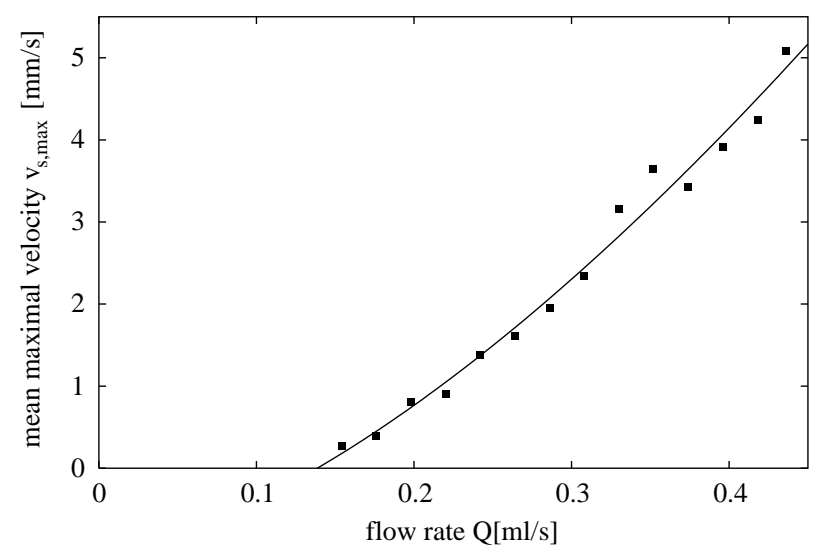

Fig. 3. - Maximal bubble velocity of convective motion as a function of flow rate. This data is averaged over downward and upward motion. The solid line is a fit to the form $v_{\mathrm{s}, \max }=a+b Q^{\delta}$ with the fit parameters $a=-1.0 \pm 0.5, b=21 \pm 3$ and $\delta=1.5 \pm 0.3$. (Tube diameter $1.1 \mathrm{~cm}$, length $30 \mathrm{~cm}$.)

reduce inertial effects. Slowly increasing the liquid flow rate $Q$ from zero increases the liquid fraction $\Phi_{1}$ of the foam in accordance with the theory of foam drainage [5], [16]. At first, only the bubbles at the very top of the foam exhibit some irregular motions, due to the input of soap solution. Eventually, we observe the onset of convective bubble motion throughout the foam.

Keeping the tube diameter fixed, we determined the onset of this convective motion as a function of the bubble radius $r$, that is the radius of a sphere with the experimentally determined bubble volume. This onset was measured at a fixed position along the vertical axis of the tube. Figure 2 shows the corresponding liquid fraction $\Phi_{1}^{\text {conv }}$ of this onset as a function of the inverse of the bubble radius. A roughly linear increase of $\Phi_{1}^{\text {conv }}$ with $r^{-1}$ is followed by a levelling off to a value of $\Phi_{1}^{\text {conv }} \simeq 0.2$.

We also determined the maximal velocity $v_{\mathrm{s}, \max }$ of the bubbles along the surface as a function of $\Phi_{1}$. Any observations of the motions in the bulk is inhibited by the diffusive light scattering of the foam. We find $v_{\mathrm{s}, \max }$ to follow a power law in $Q$ for $Q>Q^{\text {conv }}$ (corresponding to $\left.\Phi_{1}>\Phi_{1}^{\text {conv }}\right)$, as shown in fig. 3 .

In some further experiments, we incorporated a cap of bubbles of relatively large diameter at the top of the sample, which should help in suppressing any effects due to inertia or asymmetry of the input flow. In this case convection is observed with the main column of small bubbles only. We observed the same value of $\Phi_{1}^{\text {conv }}$ stated above, with some differences in the behaviour for large bubble diameter $d$, which will be analysed in further studies.

Analysis. - A full theory of the observed convective motion seems very difficult, so the following arguments concentrate on its onset. They are quite elementary, being based on simple statics and our established understanding of the rheology of foam.

A liquid foam under small loads behaves as a solid, with a finite shear modulus, and a yield stress $\Gamma$, at which the foam starts to flow like a liquid, as the individual bubbles slip over each other [12], [17]. (The same behaviour is encountered in emulsions [10], [15].) The yield stress scales with $\gamma / r$ where $\gamma$ is the surface tension and $r$ is the (average) bubble radius [18]. That is

$$
\Gamma=\frac{\gamma}{r} f\left(\Phi_{1}\right)
$$

where $f$ is a function of the liquid fraction $\Phi_{1}$ only. 
The flow of liquid in a foam column is accompanied with a shear stress, presumably proportional to the flow rate $Q$. This puts an upper limit on the flow rate which is consistent with steady flow, since the shear stress must nowhere exceed $\Gamma$.

As in Poiseuille flow of a liquid, the maximum shear stress in the foam is at its perimeter. Instability due to having reached the yield stress would occur at

$$
\Gamma=\frac{\rho g R}{2} \Phi_{1} .
$$

Here we set the shear force at the boundary equal to the weight force of the foam; $R$ is the tube radius, $g$ is the gravitational acceleration and $\rho$ is the density of the liquid.

Together with eq. (1), this suggests that data should collapse into a single line when plotted in the following way:

$$
f\left(\Phi_{1}\right)=\frac{\operatorname{Rrg\rho }}{2 \gamma} \Phi_{1} .
$$

However, such a collapse is only partially realised in practise. Moreover, the existence of wall slip at values of shear stress significantly lower than the bulk yield stress is very likely. Finally, there is preliminary evidence in our data of a dependence on the length of the column, absent from these elementary arguments. The quantitative analysis of our experimental data is therefore plainly incomplete.

Conclusion. - To our knowledge, this is for the first time that such a convective motion is described in foam, and it should be clear from the above that this is a phenomenon quite distinct from convective instabilities in ordinary fluid flows. In addition to being interesting in its own right, it should also add to our understanding of the rigidity loss in foams, a topic that still lacks a full theory.

It is clear from the results that the difficulty of creating a stable steady flow at high liquid fractions cannot be overcome simply by reducing bubble size. To probe the interesting region close to the rigidity loss transition, alternative geometries (e.g., a sloping cylinder) should be

considered. Alternatively, experiments in foams under micro-gravity [19] could be applied to this purpose.

\section{$* * *$}

This work was supported by Forbairt (Irish Science and Technology Agency) and Shell Amsterdam. SH is a TMR Fellow (European Union), Contract ERBFMBICT960970.

\section{REFERENCES}

[1] Mann W. B. and Stephen R. W. B., Philos. Mag., 15 (1933) 143.

[2] Pittet N., Rivier N. and Weaire D., Forma, 10 (1995) 65.

[3] Hutzler S., Weaire D. and Crawford R., Philos. Mag. B, 75 (1997) 845.

[4] Weaire D., Pittet N., Hutzler S. and Pardal D., Phys. Rev. Lett., 71 (1993) 2670.

[5] Weaire D., Hutzler S., Verbist G. and Peters E. A. J. F., Adv. Chem. Phys., 102 (1997) 315.

[6] Weaire D., Findlay S. and Verbist G., J. Phys. Condens. Matter, 7 (1995) L217.

[7] Hutzler S., Verbist G., Wearie D. and Van der Steen J. A., Europhys. Lett., 31 (1995) 497.

[8] Segrì P. N., Herbolzheimer E. and Chaikin P. M., Phys. Rev. Lett., 79 (1997) 2574.

[9] Bolton F. and Weaire D., Phys. Rev. Lett., 65 (1990) 3449.

[10] Lacasse M. D., Grest G. S., Levine D., Mason T. G. and Weitz D. A., Phys. Rev. Lett., 76 (1996) 3448. 
[11] Lacasse M. D., Grest G. S. and Levine D., Phys. Rev. E, 54 (1996) 5436.

[12] Durian D. J., Phys. Rev. E, 55 (1997) 1739.

[13] Princen H. M. and Kiss A. D., J. Colloid Interface Sci., 112 (1986) 427.

[14] Princen H. M. and A. D. Kiss, Langmuir, 3 (1987) 36.

[15] Mason T. G., Bibette J. and Weitz D. A., Phys. Rev. Lett., 75 (1995) 2051.

[16] Verbist G., Weaire D. and Kraynik A. M., J. Phys. Condens. Matter, 8 (1996) 3715.

[17] Hutzler S., Weaire D. and Bolton F., Philos. Mag. B, 71 (1995) 277.

[18] Weaire D. and Fortes M. A., Advan. Phys., 43 (1994) 685.

[19] Noever D. A. and Cronise R. J., Phys. Fluids, 6 (1994) 2493. 